\title{
ARTICLE
}

\section{Relaxation-depth sensitivity of In-situ $\gamma$ Spectrometry to Determine the Depth-distribution of Artificial Radionuclides in Soil}

\author{
FENG Tiancheng ${ }^{1,2 *}$, JIA Mingyan² ${ }^{2}$ CHENG Jianping ${ }^{1}$ and FENG Yuanju ${ }^{2}$ \\ ${ }^{1}$ Department of Engineering Physics, Tsinghua University, Zijing Building 0816B, Beijing 100084, China \\ ${ }^{2}$ Northwest Institute of Nuclear Technology, Xi'an, China, P.O.Box 69-1 Xi'an 710024, China
}

\begin{abstract}
The main limitation of in-situ $\gamma$ spectrometry lies in determining the depth-distribution of the artificial radionuclide in soil. Many researchers have developed methods and models for deducing the depth-distribution information from in-situ spectrum itself. Until now, such methods were studied and established as "Multiple photopeak method", "Peak-to-valley ratio method" and "Collimation or lead-plate method". This paper presented the comparative theoretical study on the relaxation-depth sensitivity. The experimental methods for Multiple photopeak, Peak-to-yalley ratio and Collimation or lead-plate are at energies of $244 \mathrm{keV}$ and $1408 \mathrm{keV} \gamma$-rays of $\mathrm{Eu}, 662 \mathrm{keV} \gamma$-ray of $\mathrm{Cs}$, $662 \mathrm{keV} \gamma$-ray of $\mathrm{Cs}$, respectively, and the In-situ object counting system was employed in Collimation or lead-plate method. Results indicated that the sequence of relaxation-depth sensitivity from the maximum to the minimum was as following: Peak-to-valley method, Multiple photopeak method, and Collimation or lead-plate method, and the sensitivity of Collimation or lead-plate method was far less than Multiple photopeak method.
\end{abstract}

KEYWORDS: in-situ y spectrometry, Multiple photopeak method, Peak-to-valley method, Collimation or lead-plate method, relaxation-depth sensitivity

\section{Introduction}

In-situ HPGe $\gamma$ spectrometry is a rapid and powerful method for the survey of radioactivity in environment. When it is used to determine the environmental radiation, initial assumptions are usually made about the depth-distribution of the radionuclide of interest in soil, in order to derive their activity concentration from the spectrum. Such assumptions can be a principal source of the uncertainty in the final results ${ }^{1,2}$. Many researchers have developed methods and models for deducing the depth-distribution information from in-situ spectrum itself. Until now, such methods were studied and established as "Peak-to-valley ratio method" ${ }^{3-9)}$, "Collimation or lead-plate method" ${ }^{10,13)}$ and "Multiple photopeak method" ${ }^{14-18)}$.

For radionuclides emitting $\gamma$-rays of several energies some parameters of depth-distribution may be determined by analysis of a single measurement based on the different absorption of $\gamma$-rays in the soil. That is due to known energy dependence of attenuation coefficient in soil. Rays with different energy are absorbed differently. Ordinarily, the depth-distribution of artificial radionuclides deposited in ground soil can be expressed approximately with an exponential function. Combining information from different energies enables us to assess the depth-distribution, which is the "Multiple photopeak method"; The deeper radionuclides distributed in soil, the more $\gamma$-rays attenuated by soil, therefore Compton effect would be strengthened, and the ratio of peak-to-valley decreases. Combining information from different ratios the depth-distribution information can be extracted, which is the "Peak-to-valley ratio method"; And using different collimators or shielding lead plates, the directional distribution of $\gamma$-rays can be determined which in turn provides information on the effective burial depth, which is the basis of the "Collimation or lead-plate method".

J MacDonald's researches ${ }^{19,20)}$ showed that the Multiple

*Corresponding Auhor, Tel No: 86-10-51537354. E-mail:

ftc07@mals.tsingha.edu.cn ${ }^{1}$

(c) Atomic Energy Society of Japan photopeak method offers the best potential sensitivity, and the lead plate method shows the least sensitivity. But until now, only J MacDonald's researches have been published to show the relaxation-depth sensitivities of these methods. And our work showed that there are some differences in the relaxation-depth sensitivities comparing to MacDonald's conclusions. Therefore, in this paper, the theoretical study was performed particularly on the relaxation-depth sensitivities for these main three methods in a comparative form.

\section{Theories}

\section{Multiple photopeak method}

For an infinite radius plane-source at depth $z_{\mathrm{i}}$ in soil, based on Beck Eq. ${ }^{1)}$ the full peak count rate in the in-situ spectrum can be calculated as following Eq. (1), and the detection efficiency $\varepsilon$ is defined as Eq. (2).

$$
\begin{aligned}
& n=\frac{A_{z} P_{\gamma} S_{0}}{2} \int_{0}^{1} \frac{F(\cos \theta)}{\cos \theta} e^{-\left(\mu_{a} h+\mu_{s} z_{i}\right) / \cos \theta} d \cos \theta \\
& \varepsilon=\frac{n}{A_{z}}=\frac{P_{\gamma} S_{0}}{2} \int_{0}^{1} \frac{F(\cos \theta)}{\cos \theta} e^{-\left(\mu_{a} h+\mu_{s} z_{i}\right) / \cos \theta} d \cos \theta
\end{aligned}
$$

Where $n$ is the full peak count rate of the $\gamma$-ray in spectrum, $\mathrm{s}^{-1} \cdot A_{\mathrm{z}}$ is the unit volume activity of the plane-source with a thickness $\Delta \mathrm{z}(\rightarrow 0)$ at depth $z_{\mathrm{i}}$ in soil, $\mathrm{Bq} / \mathrm{cm}^{3} . P_{\gamma}$ is the probability of the $\gamma$-ray emission, $\mathrm{s}^{-1} . S_{0}$ is the effective-front-area of the detector to the $\gamma$-ray, $\mathrm{cm}^{2} . F(\cos \theta)$ is the angular-response-function of the detector to the $\gamma$-ray, which is a function of the polar angle $\theta$ between the detector-symmetry-axis and a radioactive-unit-element in plane-source. $\mu_{j S}, \mu_{j A}$ are the linear attenuation coefficients of the $\gamma$-ray in soil and air respectively, $\mathrm{cm}^{-1} . h$ is the height of detector above the ground, $\mathrm{cm}$.

By the Eq. (2), $\varepsilon$ at a certain depth $z_{\mathrm{i}}$ in soil can be calculated. Calculation results show that the relationship between $\varepsilon$ and $z_{\mathrm{i}}$ corresponds with the exponential function well. The detection efficiencies of the 1st and 2th energy 
$\gamma$-rays, $\varepsilon(1)$ and $\varepsilon(2)$, can be fitted by the Eqs. (3) and (4), respectively.

$$
\begin{aligned}
& \varepsilon(1)=a e^{-b z} \\
& \varepsilon(2)=c e^{-d z}
\end{aligned}
$$

Where $a, c, b$ and $d$ all are constants. The $z$ is the depth of the plane-source in soil, $\mathrm{cm}$.

The full peak count rates of the 1 st and 2 th energy $\gamma$ rays, $n(1)$ and $n(2)$, can be expressed by Eqs. (5) and (6), respectively.

$$
\begin{gathered}
n(1)=A_{0} S \int_{0}^{\infty} e^{-\alpha z} a e^{-b z} d z=\frac{A_{0} S a}{b+\alpha} \\
n(2)=\frac{A_{0} S c}{d+\alpha}
\end{gathered}
$$

Where $A_{0}$ is the activity concentration at the soil surface, $\mathrm{Bq} / \mathrm{cm}^{3} ; \alpha$ is the reciprocal of the relaxation depth, $\mathrm{cm}^{-1}$; And the detection area $S$ is infinite, $\mathrm{cm}^{2}$.

The ratio of the full peak count rates of the 1st energy to 2th energy, $R(\alpha)$, is calculated by the following Eq. (7), which depicts the relationship between the $R(\alpha)$ and $\alpha$ concisely.

$$
R(\alpha)=\frac{n(1)}{n(2)}=\frac{a}{c} \cdot \frac{d+\alpha}{b+\alpha}
$$

\section{Collimation or lead-plate method}

In-situ object counting system (ISOCS) is employed for sensitivity analysis of "Collimation or lead-plate method", by which the detection efficiency can be calculated. For the maximization of the efficiency difference among the different shielding conditions, collimators of " $30 \mathrm{~d}-50 \mathrm{~mm}$ " and "90d-50mm 180d-50mm" were adopted.

Calculation results show that for an infinite radius plane-source at the discrete depth $z_{\mathrm{i}}$ in soil, the relationship between the $\varepsilon^{\prime}$ with collimator and $z_{\mathrm{i}}$ corresponds with the exponential function well, but there is a constant appended comparing the Multiple photopeak method above. The detection efficiencies of the 1st and 2th collimators, $\varepsilon^{\prime}(1)$ and $\varepsilon^{\prime}(2)$, can be fitted by the Eqs. (8) and (9), respectively.

$$
\begin{aligned}
& \varepsilon^{\prime}(1)=a^{\prime} e^{-b^{\prime} z}+k_{1} \\
& \varepsilon^{\prime}(2)=c^{\prime} e^{-d^{\prime} z}+k_{2}
\end{aligned}
$$

Where $a^{\prime}, c^{\prime}, b^{\prime}, d^{\prime}, k_{1}$ and $k_{2}$ all are constants.

As above, given the depth-distribution expressed with the exponential function and the detection area $S^{\prime}$, the full peak count rates of the 1 st and 2 th collimators, $n^{\prime}(1)$ and $n^{\prime}(2)$, can be expressed by Eqs. (10) and (11), respectively.

$$
\begin{gathered}
n^{\prime}(1)=A_{0} S^{\prime} \int_{0}^{\infty} e^{-\alpha z}\left(a^{\prime} e^{-b^{\prime} z}+k_{1}\right) d z=A_{0} S^{\prime}\left(\frac{a^{\prime}}{b^{\prime}+\alpha}+\frac{k_{1}}{\alpha}\right) \\
n^{\prime}(2)=A_{0} S^{\prime}\left(\frac{c^{\prime}}{d^{\prime}+\alpha}+\frac{k_{2}}{\alpha}\right)
\end{gathered}
$$

Where the detection area $S^{\prime}$ is infinite, $\mathrm{cm}^{2}$.

The ratio of the full peak count rates of the 2 th collimator to 1 st collimator, $R^{\prime}(\alpha)$, is calculated by the following Eq. (12), which depicts the relationship between the $R^{\prime}(\alpha)$ and $\alpha$.

$$
R^{\prime}(\alpha)=\frac{n^{\prime}(2)}{n^{\prime}(1)}=\frac{\left(c^{\prime}+k_{2}\right) \alpha+k_{2} d}{\left(a^{\prime}+k_{1}\right) \alpha+k_{1} b} \cdot \frac{b^{\prime}+\alpha}{d^{\prime}+\alpha}
$$

\section{Peak-to-valley ratio method}

For an infinite half-space source with exponential distribution, based on Beck Eq. the full peak count rate $\left(n^{\prime \prime}\right)$ in the in-situ spectrum can be calculated as following Eq. (13).

$$
n^{\prime \prime}=\frac{A_{0} P_{\gamma} S_{0}}{2} \int_{0}^{1} \frac{F(\cos \theta)}{\left(\mu_{s}+\alpha \cos \theta\right)} \cdot e^{-\mu_{a} h / \cos \theta} d \cos \theta
$$

The probability $\left(P_{1}\right)$ of single scattering rays emitting from soil can be expressed as the Eq. ${ }^{7)}(14)$.

$$
\begin{aligned}
P_{1} & =\int_{0}^{r-h / \cos \theta} k \mu_{s} e^{-\mu_{s} x} d x \cdot e^{-\mu_{s}(r-h / \cos \theta-x)} e^{-\mu_{a} h / \cos \theta} \\
& =k \mu_{s}(r-h / \cos \theta) e^{-\mu_{s}(r-h / \cos \theta)-\mu_{a} h / \cos \theta}
\end{aligned}
$$

Where $r$ is the distance from radioactive unit-volume-element to detector, $\mathrm{cm}$. $k$ is the fraction of interacted photons scattered into the 'valley'.

Based on the Eq. (14), the net valley count rate $(C)$ due to the interaction only with soil in the in-situ spectrum, can be calculated as following Eq. (15).

$$
C=\frac{A_{0} P_{\gamma} S_{0} k \mu_{s}}{2} \int_{0}^{1} \frac{F(\cos \theta)}{\left(\mu_{s}+\alpha \cos \theta\right)^{2}} \cdot e^{-\mu_{a} h / \cos \theta} d \cos \theta
$$

Therefore, the peak-to-valley ratio $\left(R^{\prime \prime}(\alpha)\right)$ can be calculated by the following Eq. (16).

$$
R^{\prime \prime}(\alpha)=\frac{n^{\prime \prime}}{C}=\frac{1}{k \mu_{s}} \cdot \frac{\int_{0}^{1} \frac{F(\cos \theta)}{\left(\mu_{s}+\alpha \cos \theta\right)} \cdot e^{-\mu_{a} h / \cos \theta} d \cos \theta}{\frac{F(\cos \theta)}{\left(\mu_{s}+\alpha \cos \theta\right)^{2}} \cdot e^{-\mu_{a} h / \cos \theta} d \cos \theta}
$$

\section{Sensitivities analysis}

\section{Method parameters}

(1) Multiple photopeak method

Given the soil density $1.6 \mathrm{~g} / \mathrm{cm}^{3}$ and a GMX HPGe detector, the detection efficiencies $(\varepsilon)$ of $244 \mathrm{keV}$ and $1408 \mathrm{keV} \gamma$-rays of ${ }^{152} \mathrm{Eu}$ are calculated by the Eq. (2), and show as following Fig.1, and the fitting parameters ( $a, c, b$ and $d$ ) are listed in the table 1 also.

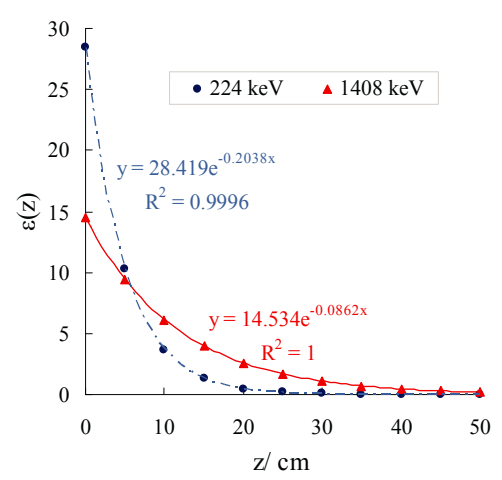

Fig.1 The detection efficiencies $\varepsilon$ of $244 \mathrm{keV}$ and $1408 \mathrm{keV}$ $\gamma$-rays of ${ }^{152} \mathrm{Eu}$.

Table 1 Parameters of Multiple photopeak method.

\begin{tabular}{c|c|c|c|c}
\hline Parameters & $a$ & $b\left(\mathrm{~cm}^{-1}\right)$ & $c$ & $d\left(\mathrm{~cm}^{-1}\right)$ \\
\hline Values & 28.419 & 0.2038 & 14.534 & 0.0862 \\
\hline
\end{tabular}

(2) Collimation or lead-plate method

As above, the detection efficiencies $\left(\varepsilon^{\prime}\right)$ of $662 \mathrm{keV} \gamma$-rays of ${ }^{137} \mathrm{Cs}$ with $30 \mathrm{~d}-50 \mathrm{~mm}$ and $90 \mathrm{~d} \sim 180 \mathrm{~d}-50 \mathrm{~mm}$ collimators are calculated using ISOCS-software, and show as following Fig.2, and the fitting parameters $\left(a^{\prime}, c^{\prime}, b^{\prime}, d^{\prime}, k_{1}\right.$ and $\left.k_{2}\right)$ are listed in the table 2 also. 
Table 2 Parameters of Collimation or lead-plate method.

\begin{tabular}{c|c|r|c|c|c|c}
\hline Par. & $a^{\prime}$ & $b^{\prime}\left(\mathrm{cm}^{-1}\right)$ & $k_{1}$ & $c^{\prime}$ & $d^{\prime}\left(\mathrm{cm}^{-1}\right)$ & $k_{2}$ \\
\hline Val. & 0.9829 & 0.1914 & 0.01074 & 0.9942 & 0.2239 & 0.00418 \\
\hline
\end{tabular}

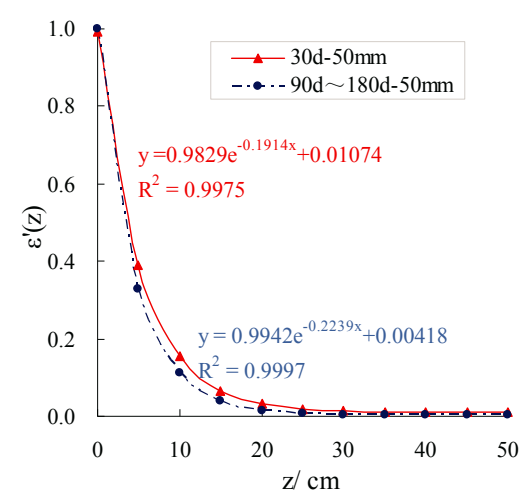

Fig. 2 The detection efficiencies $\varepsilon^{\prime}$ of $30 \mathrm{~d}-50 \mathrm{~mm}$ and $90 \mathrm{~d} \sim 180 \mathrm{~d}-50 \mathrm{~mm}$ collimators.

(3) Peak-to-valley ratio method

Given $F(\cos \theta)=1$, and $k$ an arbitrary value that couldn't affect the analysis results. The peak-to-valley ratio $\left(R^{\prime \prime}(\alpha)\right)$ was calculated by the Eq. (16), and show as following Fig.3.

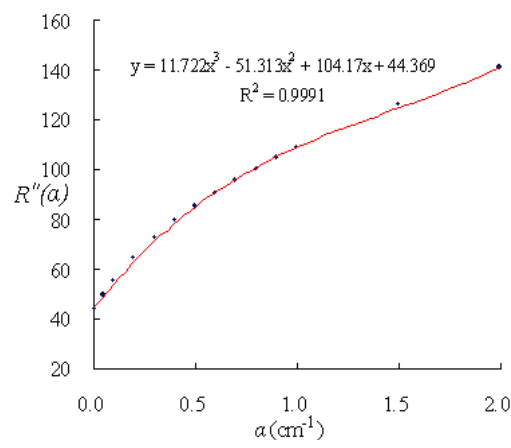

Fig.3 Relationship between $R^{\prime \prime}(\alpha)$ and $\alpha$ (the reciprocal of the relaxation depth)

\section{Sensitivities comparison}

Based on the method parameters, the values of each method-ratio above were calculated by the Eqs. (7), (12) and (16). For comparing the potential sensitivity of each method, the values of each method-ratio were normalized to 1 at a particular value of the parameter $\alpha=0 \mathrm{~cm}^{-1}$. The choice of this normalization point is completely arbitrary but, for visual simplicity, was chosen to be at one extent of the realistic range. The relative relaxation-depth sensitivity graphs were given as following Fig.4.

As can be seen from these graphs, while $0<\alpha<0.3 \mathrm{~cm}^{-1}$ (corresponding to the radioactive distribution

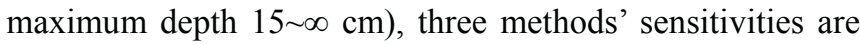
all relative high, and the sequence from the high to the low can be expressed as following: "Peak-to-valley" $\approx$ "Multiple photopeak" >> "Collimated or lead plate"; while $\alpha>0.3 \mathrm{~cm}^{-1}$ (corresponding to the radioactive distribution maximum depth $0 \sim 15 \mathrm{~cm}$ ), Peak-to-valley method shows the greatest variation in method-ratio, offering the best potential sensitivity, and Collimation or lead-plate method shows the least even neglectable variation in method-ratio, therefore the sequence of relaxation-depth sensitivity from the high to the low should be express as following: "Peak-to-valley" >> "Multiple photopeak" > "Collimated or lead plate"; In general, the sequence of relaxation-depth sensitivity from the maximum to the minimum was as following: Peak-to-valley method, Multiple photopeak method, and Collimation or lead-plate method, and the sensitivity of Collimation or lead-plate method was far less than Multiple photopeak method.

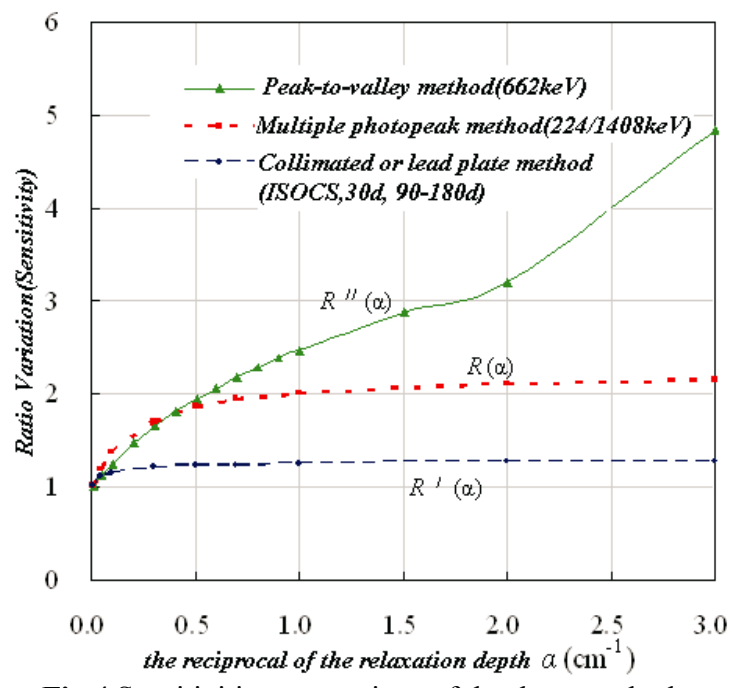

Fig.4 Sensitivities comparison of the three methods.

Comparing with the J MacDonald's results ${ }^{19,20)}$, there are some differences in the sensitivity relationship between Peak-to-valley ratio method and Multiple photopeak method. J MacDonald's research showed that the sensitivity of Peak-to-volley ratio is less than Multiple photopeak. It is because that J MacDonald's research adopted 32keV X-rays absorbed by soil seriously, but our work employed the least energy at $224 \mathrm{keV}$. In practice, $32 \mathrm{keV} \mathrm{X}$-rays is unseemliness in the field measurement due to the complicated background disturbance. In other ways, while the radionuclide distributed on the ground surface $(z \rightarrow 0)$, the Peak-to-valley ratio would tend to become the infinity, but the ratio of Multiple photopeak method is a limited constant only. Therefore, the verdict that relaxation-depth sensitivity of Peak-to-volley ratio is higher than Multiple photopeak should be rational.

To Collimation or lead-plate method, we adopted the most prime collimator-combination (30d-50mm, and $90 \sim$ $180 \mathrm{~d}-50 \mathrm{~mm}$ ) with the greatest variation in method-ratio. However, the changing range of the method-ratio is very small, which shows that the competence of Collimation or lead-plate method to determine the depth-distribution is very limited. And Robert's researches ${ }^{21)}$ show that Collimation or lead-plate method only can be used to distinguish between the infinite homogeneous distribution and the surface distribution, which is according well with our results.

\section{Conclusions}

By the theoretical study, the sensitivity comparison of three main methods for the depth-distribution measurement of radionuclides in soil has been performed. The 
experimental methods for Multiple photopeak, Peak-to-valley ratio and Collimated or lead plate are at the energy of $244 \mathrm{keV}$ and $1408 \mathrm{keV} \gamma$-rays of ${ }^{152} \mathrm{Eu}, 662 \mathrm{keV}$ $\gamma$-ray of ${ }^{137} \mathrm{Cs}, 662 \mathrm{keV} \gamma$-ray of ${ }^{137} \mathrm{Cs}$, respectively, and the ISOCS was employed in Collimation or lead-plate method. Results show that the sequence of relaxation-depth sensitivity from the maximum to the minimum was as following: Peak-to-valley method, Multiple photopeak method, and Collimation or lead-plate method, and the sensitivity of Collimation or lead-plate method was far less than Multiple photopeak method.

Peak-to-valley ratio method can be applied to the arbitrary

\section{References}

1) Harold L. Beck, Joseph Decampo, Carl Gogolak. In situ $\mathrm{Ge}(\mathrm{Li})$ and $\mathrm{NaI}(\mathrm{Tl})$ gamma-ray spectrometry. HASL-258, (1972).

2) A Likar, G Omahen, T Vidmar, R Martinčič. Method to determine the depth of Cs-137 in soil from in-situ gamma-ray Spectrometry. J. Phys. D: Appl. Phys., 17,2825 (2000).

3) Andrew N. Tyler. Monitoring anthropogenic radioactivity in salt marsh environments through in situ gamma-ray spectrometry. Journal of Environmental Radioactivity, 45,235(1999).

4) Andrew N. Tyler. High accuracy in situ radiometric mapping. Journal of Environmental Radioactivity, 72,195(2004).

5) Thomas Hjerpe, Christer Samuelsson. Accounting for the depth-distribution of ${ }^{137} \mathrm{Cs}$ in on-line mobile gamma spectrometry through primary and forward-scattered photons. Radiat Environ Biophys, 41,225(2002).

6) F. Gering, U. Hillmann, P. Jacob, G. Fehrenbacher. In situ amma-spectrometry several years after deposition of radiocesium II. Peak-to-valley Method. Radiat Environ Biophys, 37,283(1998).

7) Zombori P, Andrasi A and Nkmeth I. A New Method for the Determination of Radionuclide Distribution in the Soil by In-Situ Gamma-Ray Spectrometry. Report KFKI, 1992-20/K, Central Research Institute for Physics, Hungarian Academy of Sciences, Budapest, (1992).

8) A. N. Tyler, D. C. W. Sanderson, E. M. Scott. Estimating and Accounting for ${ }^{137} \mathrm{Cs}$ Source Burial through In-Situ Gamma Spectrometry in Salt Marsh Environments. J. Environ. Radioactivity,33[3],195(1996).

9) Q. He, D.E. Walling. Calibration of a field-portable gamma detector to obtain in situ measurements of the ${ }^{137} \mathrm{Cs}$ inventories of cultivated soils and floodplain sediments. Applied Radiation and Isotopes, 52,865(2000).

10) M. Korun, A. Likar, M. Lipoglavšek, R. Martinčič, B.Pucelj. In-situ measurement of Cs distribution in the soil. Nuclear Insteuments Methods in Physics Research B, 93,485(1994).

11) Sabina Markelj, Matej Lipoglavšek. In-situ measurement of Cs distribution in the soil. J. Stefan Institute, University of Ljubljana, Ljubljana, Slovenia. (2004).

12) Roland R. Benke, Kimberlee J. Kearfott. An improved in situ $\gamma$ emitter radionuclide. Multiple photopeak one could be adopted for the radionuclide emitting at least two energy $\gamma$-rays. These two methods are more sensitive and practicable than Collimation or lead-plate method to determine the depth distribution of radionuclides in soil, and Peak-to-valley ratio method is better than Multiple photopeak method, in general. To Collimation or lead-plate method, not only it's least sensitivity, but also the potential fatal error due to radioactive unhomogeneity along the landscape-orientation, therefore it's competence to determine the depth-distribution is very limited and debatable.

method for determining depth-distributions of gamma-ray emitting radionuclides. Nuclear Instruments and Methods in Physics Research A, 463,393(2001).

13) Roland R. Benke, Kimberlee J. Kearfott. Demonstration of a collimated in situ method for determining depth-distributions using $\gamma$-ray spectrometry. Nuclear Instruments and Methods in Physics Research A, 482,814(2002).

14) K. Rybacek, P. Jacob, R. Meckbach. In-situ determination of deposited radionuclide activities: Improved method using derived depth-distributions from the measurement photon spectrum. Health Physics, 62[6],519(1992).

15) Christopher P. Oertel, John R. Giles, Kenneth C. Thompson, and Richard P. Wells. In situ depth profiling of ${ }^{137} \mathrm{Cs}$ contamination in soils at the IDAHO National Engineering and Environmental Laboratory. Health Phys. 87[6],664(2004).

16) Severin Thummerer, Peter Jacob. Determination of depth-distributions of natural radionuclides with in situ gamma-ray spectrometry. Nuclear Instruments and Methods in Physics Research A,416,161(1998).

17) M. Korun, R. Martinčič, B. Pucelj. In-situ measurements of the radioactive fallout deposit. Nuclear Instruments and Methods in Physics Research A, 300,611(1991).

18) T.C. Feng, M.Y. Jia, B. Long, C.Y. Su, R. Wu, J.P. Cheng. Delaminated method to determine the depth-distribution of ${ }^{152} \mathrm{Eu}$ in soil by in-situ HPGe $\gamma$ spectrometry. Nuclear Instruments and Methods in Physics Research A,597,192(2008).

19) J MacDonald, C J Gibson, P J Fish and D J Assinder. A theoretical comparison of methods of quantification of radioactive contamination in soil using in situ gamma spectrometry. J. Radiol. Prot., 17[1],3(1997).

20) J MacDonald, C J Gibson, P J Fish, D J Assinde. An Experimental Comparison of In-Situ Gamma Spectrometric Methods for Quantifying Cs-137 Radioactive Contamination in the Ground. IEEE TRANSACTIONS ON NUCLEAR SCIENCE, 46[3],429(1999).

21) Robert R. Finck. High Resolution Field Gamma Spectrometry and its application to Problems in Environmental Radiology (Doctoral Dissertation). Departments of Radiation Physics, Malmö and Lund Lund University (Malmö, Sweden). (1992). 